\title{
25. GEOCHEMISTRY OF CARBON: DEEP SEA DRILLING PROJECT LEGS 58 AND 59
}

\author{
Karl S. Schorno, Phillips Petroleum Company, Bartlesville, Oklahoma
}

\section{INTRODUCTION}

I obtained 68 quarter sections of cores from the JOIDES Organic Geochemistry Panel for studying type, distribution, and stages of organic diagenesis of sedimentary organic matter in the West Philippine and Parece Vela basins and Mariana Trough area (Figure 1). The present chapter compares (1) 11 geochemical parameters used to determine organic source and its stage of genesis within the 9 site locations in this study area and (2) compares these 11 with the same parameters reported from Leg 56, outer trench slope of the Japan Trench, and Leg 60, Mariana Trough and Trench (Schorno, in press a, b). Even though these sediments are considered pelagic, the organic content in most of the core sections appears to be hemipelagic. The sedimentary organic matter in these cores is believed to be in an early stage of diagenesis. Both conclusions are based primarily on the $n$-alkane distribution within the organic matter. This particular parameter, I note later, has a major weakness. As Hunt (Hunt, 1979) and I (in press b) observed, marine organisms synthesize $n$-alkanes with distributions containing neither odd nor even preferences. Thus those sediments that did contain $n$-alkane distributions with $\overline{O E P}$ near 1, suggesting a late stage of catagenesis, may in actuality be immature marine sediments.

\section{SAMPLING AND STUDY PROCEDURES}

The sampling procedure used in this study is shown schematically in Figure 2. The frozen quarter sections were crushed to $\leq 150 \mu \mathrm{m}$ (100 mesh) and an aliquot analyzed for organic and inorganic carbon and nitrogen by oxidative-combustion and Kjeldahl procedures, respectively. The crushed core was soxhlet-extracted with methylene chloride and the solvent removed at $45^{\circ} \mathrm{C}$ at atmospheric pressure. Because of the paucity of extract in these organic-lean cores, the analysis of the extract is limited to a capillary gas chromatography and stable carbon isotope determinations.

\section{RESULTS AND DISCUSSION}

The organic geochemical data appear in Tables 1 and 2 and the average values for each set of data for each site in Table 3. The following sections will be broken into a discussion of each of five organic chemical properties-the organic content, $n$-alkane content, ratio of pristane to phytane, stable carbon isotopic compositions of both bitumen and kerogen fractions, and the organic nitrogen content-of these cores. Finally, the average values of 11 geochemical properties of these cores are compared with similar averaged values from sediments from the Mariana Trough and Trench recovered during Leg 60 and the outer trench slope from the Japan Trench recovered during Leg 56.

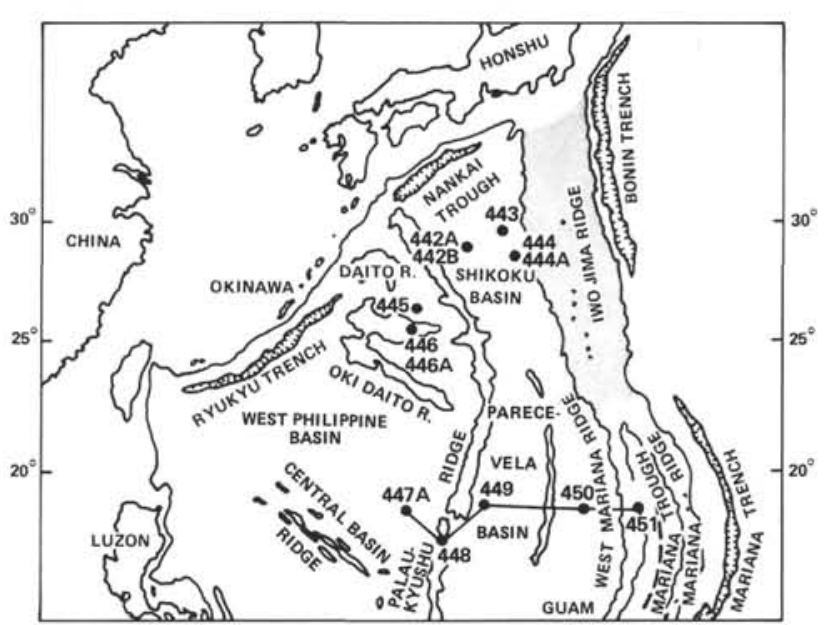

Figure 1. Location of sites from which samples were obtained for geochemical study.

\section{Organic Content}

The organic content of the 68 cores obtained for this study is low, averaging $0.12 \%$ and ranging from $0.01 \%$ to $0.33 \%$. Variations in the values of organic carbon within each site or among sites may be significant or may reflect only the precision of this particular measurement. We can reproduce the total organic carbon (TOC) content to $0.01 \%$. However, the values given in Table 1 are based on a single measurement. There is a possibility that the sample is slightly inhomogeneous, which could account for slight fluctuation in the TOC. Even though these values are low, there is an apparent trend in the organic carbon content in Holes 442A, $442 \mathrm{~B}, 443,444$, and $444 \mathrm{~A}$ in the Shikoku Basin, and in Hole 445, in the small basin in the Daito Ridge. The trend appears to be real in that the organic carbon content of the Pleistocene sediments at these locations is slightly higher than that of the lower units. Moreover, the organic carbon content of the remaining cores from the study area is relatively constant and near the average value for all of the sites.

The bitumen content reflects the low TOC content within each site. Because the amount of TOC is less than $3300 \mathrm{ppm}(0.33 \%)$, the bitumen content of course cannot be greater. The bitumen content ranges from a low of $3 \mathrm{ppm}$ (Sample 449-13-4) to a high of $440 \mathrm{ppm}$ (Sample $450-2-5$ ). TOC content for these two samples is 2200 ppm and $3200 \mathrm{ppm}$, respectively. Of the 68 cores studied, only 4 contain over $100 \mathrm{ppm}$ bitumen. Of the remaining 64 cores, the average bitumen content is 24 ppm. 


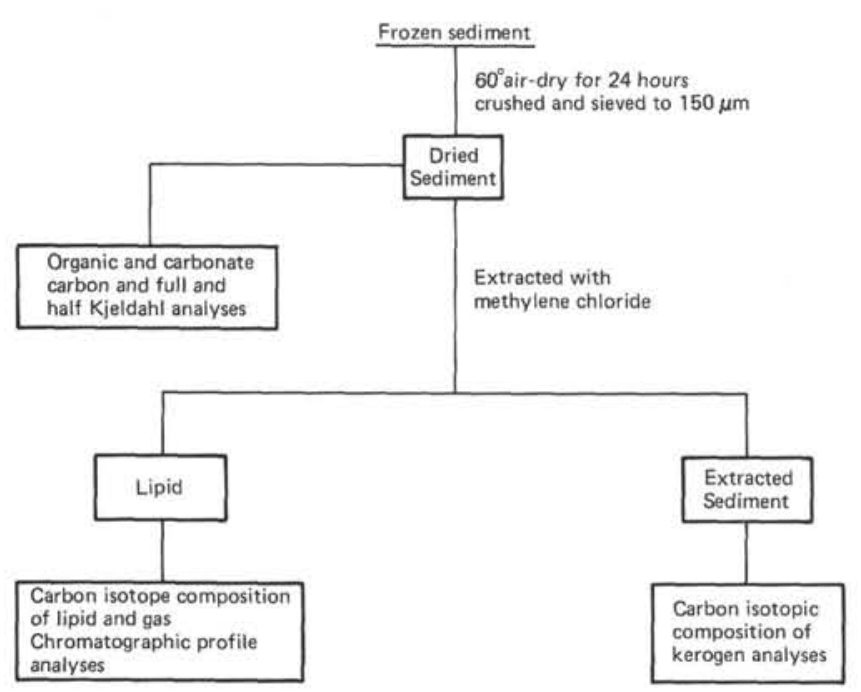

Figure 2. Flow sheet for the separation and characterization of organic matter from DSDP cores from Legs 58 and 59.

The portion of the TOC that is bitumen carbon varies from a high of 31.6 (Sample 444A-9-3) to a low of $0.11 \%$ (Sample $445-42-5$ ). Only 10 out of 67 of the values given in Table 1 are greater than $10 \%$. Of these 10 , the TOC content of 9 is less than $0.05 \%$. Because the content is so low, only a trace amount of contaminant, such as soluble sulfur, can have had a signficant effect on this value. Thus the high percentage of bitumen in these few cores may be suspect. The average percentage of bitumen in the remaining cores is 1.59 , a typical value for most bitumen fractions of DSDP-IPOD cores that contain immature organic matter.

\section{n-Alkane Content}

The discussion of the $n$-alkane content is divided into two sections. The first deals with the study of the relative abundance of marine versus terrestrial organic matter within the cores as given in the $S$-value $S=\left(n-\mathrm{C}_{21}+n-\mathrm{C}_{22}\right) /\left(n-\mathrm{C}_{28}+n-\mathrm{C}_{29}\right)$ (Philippi, 1974). According to Philippi, the range of $S$-values for crudes and their terrigenous rocks is from 0.6 to 1.2 and for marine rocks from 1.5 to 5 . The second describes the difficulty in using $n$-alkane distributions for determining maturation stages of purely marine sediments. A well-accepted maturation parameter is the ratio of odd to even $n$-alkanes in the range from $n-\mathrm{C}_{23}$ to $n$ - $\mathrm{C}_{35}$. This ratio is described by a mathematical expression $(\overline{O E P})$ developed by Scalan and Smith (1970):

$$
\overline{O E P}=\left[\frac{C_{i}+6 \mathrm{C}_{i+2}+\mathrm{C}_{i+4}}{4 \mathrm{C}_{i+1}+4 \mathrm{C}_{i+3}}\right](-1)^{i+1} .
$$

In this case, organic matter in the major stage of genesis, the catagenesis stage (Tissot and Welte, 1978), is described by an $\overline{O E P}$ of less than 1.3. Sedimentary organic matter containing $\overline{O E P}$ values greater than 1.3 are considered immature-that is, in the diagenetic stage. As I point out in the next section, both $S$ and
$\overline{O E P}$ are suspect for the particular usage outlined in the foregoing for pure marine sediments.

The amount of terrigenous organic matter within these sediments as inferred by $S$ less than 1 is high in 36 of the 68 cores. Of the 68 cores, 25 contained $S$ greater than 1 but less than 1.5 , and 7 contained $S$ values greater than 1.5. In many cases where $S$ is low, the $n$-alkane distribution from $n-C_{15}$ to $n-C_{35}$ has a minimum of approximately $n-C_{22}$. In several cases the maximum at $n-\mathrm{C}_{18}$ is larger than the second maximum at $n-\mathrm{C}_{28}$, suggesting that the Philippi selection (1974) for the $n$-alkanes for representing marine-derived organic matter was poor.

According to the maturation interpretation placed upon $\overline{O E P}$ values, 45 of the 65 cores analyzed are in the diagenetic stage of maturation $(\overrightarrow{O E P}>1.3)$. The remaining 20 sediments are in the catagenetic to metagenetic stages of maturation $(\overline{O E P}<1.3)$. In the latter case there is some evidence of igneous intrusions in the study area that could explain the higher stages of maturation. Because these are limited to at most two of these sediments, I believe that the low values are due to the occurrence of $n$-alkanes with neither odd nor even preferences-that is, they are included in the original sedimentary organic matter. Two examples are reported in the literature of immature open marine sediments containing $\overline{O E P}$ values of near 1 . These are the Cariaco Trench sediments reported by Hunt (1979) and the Mariana Trough and Trench sediments reported by me (Schorno, in press b). Presumably, marine organisms can synthesize high molecular weight $n$-alkanes that have $\overline{O E P}$-values near 1 .

\section{Pristane to Phytane Ratio (Pr/Ph)}

In the past, the ratio of pristane to phytane $(\mathrm{Pr} / \mathrm{Ph})$ has been used to distinguish marine from terrigenous organic matter or, more accurately, from organic matter derived in an oxidizing versus reducing environment of deposition (Lijmbach, 1975; Powell and McKirdy, 1973). By empirical observation it was noted that marine-derived organic matter contained a predominance of phytane $(\mathrm{Pr} / \mathrm{Ph}$ less than 1$)$ and that for terrigenous-derived organic matter $\mathrm{Pr} / \mathrm{Ph}$ is greater than 1.5. Of the 68 samples studied, 60 contain $\mathrm{Pr} / \mathrm{Ph}$ less than 1 , indicating either a predominance of marine organic matter or a reducing environment of deposition.

\section{Carbon Isotopic Composition of the Bitumen and Kerogen Fractions ${ }^{1}$}

The number of samples for which carbon isotopic composition determinations are reported is small for the bitumen fraction because of limited sampling, whereas all but six values are reported for the kerogen. Of the

\footnotetext{
${ }^{1}$ Carbon isotopic composition is reported herein by $\delta^{13} \mathrm{C}_{P D B}$. $P D B$ is the carbonate standard from the Pee Dee Formation in North Carolina.

$$
{ }_{8}^{13} \mathrm{C}_{P D B}=\left[\frac{{ }^{13} \mathrm{C} /{ }^{12} \mathrm{C} \text { sample }-{ }^{13} \mathrm{C} /{ }^{12} \mathrm{C} P D B}{{ }^{13} \mathrm{C} / 12 \mathrm{C}_{P D B}}\right] \times 1000
$$
}


Table 1. Geochemical data from frozen core sections from DSDP Legs 58 and 59.

\begin{tabular}{|c|c|c|c|c|c|c|c|c|c|c|c|c|c|c|c|}
\hline & & & & & Carbc & onate & Organic & Carbon & & $\overline{O E P}$ & $\begin{array}{r}\text { Cas } \\
\text { Compo }\end{array}$ & $\begin{array}{l}\text { bon Isotop } \\
\text { sition }(\delta)^{1}{ }^{3} \mathrm{C}\end{array}$ & $P D B)$ & $\begin{array}{r}\text { Kjeld } \\
\text { Nitrogen }\end{array}$ & $\begin{array}{l}\text { alh } \\
\text { (ppm) }\end{array}$ \\
\hline Core & Section & $\begin{array}{l}\text { Interval } \\
\text { (cm) }\end{array}$ & Chronostratigraphy & $\begin{array}{l}\text { Sub-bottom } \\
\text { Depth }(m)\end{array}$ & $\begin{array}{l}\text { Carbon } \\
\text { (wt. \%) }\end{array}$ & $\begin{array}{c}\text { As } \\
\mathrm{CaCO}_{3} \\
\text { (wt. } \% \text { ) }\end{array}$ & $\begin{array}{l}\text { Total } \\
\text { (wt. \%o) }\end{array}$ & $\begin{array}{c}\text { Bitumen } \\
(\mathrm{ppm})\end{array}$ & $\frac{\text { Bitumen }}{\begin{array}{c}\text { TOC } \\
\text { (wt. \%) }\end{array}}$ & & Bitumen & Kerogen & $\begin{array}{l}\text { Diff. } \\
B-K\end{array}$ & $\begin{array}{c}\text { Ammonium } \\
\text { Nitrogen } \\
\text { (half) }\end{array}$ & $\begin{array}{l}\text { Organic N } \\
\text { (full-half) }\end{array}$ \\
\hline & & & & & & West-ce & entral part & of the Shikc & ku Basin & & & & & & \\
\hline $\begin{array}{l}\text { Leg } 58 \\
\text { Hole }\end{array}$ & $442 A, \mathrm{Ur}$ & & & & & & & & & & & & & & \\
\hline 2 & 3 & 93-118 & upper Pleistocene & 13.8 & 0.38 & 3.15 & 0.23 & 28 & 1.73 & 3.1 & -26.4 & -23.3 & -3.1 & 84 & 516 \\
\hline 7 & 3 & $100-125$ & upper Pleistocene & 61 & 0.63 & 5.2 & 0.30 & 17 & 0.58 & 2.3 & - & -11.8 & - & 45 & 662 \\
\hline 14 & 2 & $100-125$ & upper Pleistocene & 127.5 & 0.28 & 2.33 & 0.15 & 34 & 2.25 & 1.7 & -27.0 & -23.0 & -4.0 & 48 & 595 \\
\hline Hole & $442 \mathrm{~A}, \mathrm{Un}$ & it II, Sub-ur & nit $11 \mathrm{~A}$ & & & & & & & & & & & & \\
\hline 19 & 2 & $100-125$ & upper Pliocene & 173.5 & 0.32 & 2.68 & 0.03 & 5 & 1.88 & 3.0 & - & -24.2 & - & 30 & 494 \\
\hline 19 & 2 & $125-150$ & upper Pliocene & 174.7 & 0.11 & 0.91 & 0.33 & 7 & 0.34 & 2.0 & -25.8 & -25.7 & -0.1 & 26 & 417 \\
\hline Hole & $442 \mathrm{~A}, \mathrm{Un}$ & it III, Sub-u & init $111 \mathrm{~A}$ & & & & & & & & & & & & \\
\hline 23 & 2 & $100-125$ & & 211.8 & 0.25 & 0.04 & 0.06 & 111 & 19.91 & - & -26.5 & -25.6 & -0.9 & 16 & 477 \\
\hline 23 & 2 & $125-150$ & & 213 & 0.34 & 2.83 & 0.05 & 35 & 7.19 & 2.2 & -26.4 & -27.0 & +0.6 & 36 & 478 \\
\hline 28 & 2 & $100-125$ & middle Miocene & 259 & 0.03 & 0.28 & 0.29 & 20 & 0.70 & 1.7 & -30.7 & -22.1 & -8.6 & 38 & 541 \\
\hline 28 & 2 & $125-150$ & middle Miocene & 260.5 & 0.08 & 0.67 & 0.01 & 23 & 23.3 & 1.9 & -27.5 & -26.4 & -1.1 & 23 & 343 \\
\hline Hole & 442B, Un & it III, Sub-u & nit $111 \mathrm{~B}$ & & & & & & & & & & & & \\
\hline 2 & 3 & $100-125$ & lower Miocene & 281 & 0.19 & 1.58 & 0.01 & 13 & 12.8 & 2.1 & - & -27.9 & - & 12 & 30 \\
\hline & & & & & & East-cer & entral part & f the Shiko & ku Basin & & & & & & \\
\hline Hole & 443, Unit & IV, Sub-un & it IVA & & & & & & & & & & & & \\
\hline 23 & 3 & $100-125$ & upper Miocene & 210.5 & 0.19 & 1.58 & 0.04 & 24 & 6 & 1.2 & -26.5 & - & - & 35 & 455 \\
\hline 31 & 3 & $100-125$ & upper Miocene & 291 & 0.22 & 1.83 & 0.03 & 23 & 7.73 & 1.8 & -26.6 & -25.8 & -0.8 & 33 & 340 \\
\hline 34 & 2 & $100-125$ & upper Miocene & 318 & 1.26 & 10.49 & 0.24 & - & - & - & - & -28.2 & - & 24 & 266 \\
\hline Hole & 444, Unit & 1, Sub-unit & IA & & & & & & & & & & & & \\
\hline 2 & 4 & $94-119$ & upper Pleistocene & 11.5 & 0.44 & 3.67 & 0.31 & 35 & 1.13 & 1.5 & -30.7 & -22.8 & -7.9 & 65 & 649 \\
\hline Hole & 444, Unit & & & & & & & & & & & & & & \\
\hline 7 & 5 & $94-119$ & upper Pliocene & 60.5 & 0.22 & 1.83 & 0.01 & 30 & 30.1 & 3.2 & -25.8 & -25.8 & 0.0 & 14 & 187 \\
\hline Hole & 444A, Un & it III, Sub-u & Init IIIA & & & & & & & & & & & & \\
\hline 1 & 4 & $100-125$ & upper Miocene & 87.5 & 0.23 & 1.92 & 0.01 & 10 & 10.3 & 1.5 & -30.7 & -27.1 & -3.6 & 28 & 247 \\
\hline Hole & $444 \mathrm{~A}, \mathrm{Un}$ & it III, Sub-u & nit IIIB & & & & & & & & & & & & \\
\hline 6 & 4 & $71-96$ & upper Miocene & 134.7 & 0.20 & 1.67 & 0.01 & 10 & 10 & 1.9 & -26.0 & -26.9 & +0.9 & 33 & 390 \\
\hline 9 & 3 & $90-115$ & upper Miocene & 162 & 0.70 & 5.83 & 0.01 & 32 & 31.6 & 1.4 & -27.2 & -26.3 & -0.9 & 31 & 257 \\
\hline Hole & $444 \mathrm{~A}, \mathrm{Un}$ & it IV, Sub-u & nit IVB & & & & & & & & & & & & \\
\hline 14 & 3 & $100-125$ & middle Miocene & 209.3 & 0.59 & 4.92 & 0.01 & 15 & 14.5 & - & -25.7 & - & - & 13 & 155 \\
\hline Hole & 444A, Un & it IV, Sub-u & nit IVC & & & & & & & & & & & & \\
\hline 22 & 5 & $100-125$ & lower-middle Miocene & 269.3 & 0.23 & 1.92 & 0.01 & 25 & 25.4 & 0.9 & -27.2 & -27.5 & +0.3 & 22 & 192 \\
\hline & & & & & & & Small Basi & 1 in the $\mathrm{Da}$ & to Ridge & & & & & & \\
\hline Hole & 445, Unit & I, Sub-unit & & & & & & & & & & & & & \\
\hline 1 & 4 & $94-119$ & upper Pleistocene & 4.0 & 5.32 & 44.37 & 0.23 & 119 & 5.25 & & -27.3 & -21.9 & -5.4 & 27 & 361 \\
\hline 1 & 4 & $119-144$ & upper Pleistocene & 5.5 & 5.94 & 49.50 & 0.29 & 111 & 3.89 & 1.5 & - & -27.5 & - & 24 & 294 \\
\hline 6 & 5 & $94-119$ & upper Pliocene & 53.5 & 5.44 & 45.33 & 0.05 & 19 & 3.76 & 1.7 & - & -25.0 & - & 33 & 240 \\
\hline Hole & 445, Unit & 1, Sub-unit & IB & & & & & & & & & & & & \\
\hline 11 & 2 & $100-125$ & upper Pliocene & 96.5 & 0.19 & 1.58 & 0.01 & 17 & 0.17 & 1.6 & -27.0 & -24.7 & -2.3 & 22 & 173 \\
\hline Hole & 445, Unit & II, Sub-unit & IIA & & & & & & & & & & & & \\
\hline 17 & 1 & $90-115$ & upper Miocene & 152 & 0.19 & 1.58 & 0.01 & 21 & 20.6 & 4.2 & -27.0 & -23.8 & -3.2 & 11 & 160 \\
\hline 23 & 4 & $0-25$ & upper Miocene & 212.5 & 0.96 & 8.00 & 0.01 & 17 & 0.17 & 4.5 & -26.9 & -24.8 & -2.1 & 15 & 193 \\
\hline 27 & 4 & $94-119$ & upper Miocene & 251.5 & 6.63 & 55.25 & 0.01 & 22 & 0.22 & 2.2 & -25.3 & -25.9 & +0.6 & 23 & 252 \\
\hline Hole & 445, Unit & 11. Sub-unit & IIB & & & & & & & & & & & & \\
\hline 32 & 2 & $90-115$ & lower-middle Miocene & 296 & 12.00 & 100.00 & 0.02 & 11 & 5.60 & & -24.7 & -27.3 & +2.4 & 15 & 105 \\
\hline Hole & 445, Unit & II, Sub-unit & IIC & & & & & & & & & & & & \\
\hline 37 & 4 & $90-115$ & upper Miocene & 346 & 9.43 & 78.55 & 0.15 & 1 & 0.07 & 1.3 & - & -27.7 & - & 21 & 178 \\
\hline 42 & 5 & $100-125$ & upper Oligocene & 395 & 10.24 & 85.33 & 0.01 & 11 & 0.11 & 1.2 & -26.2 & -26.1 & -0.1 & 10 & 110 \\
\hline 47 & 4 & $100-125$ & upper Oligocene & 441.5 & 4.63 & 38.58 & 0.01 & 13 & 0.13 & 1.0 & -26.0 & -25.3 & -0.7 & 10 & 70 \\
\hline 52 & 4 & $100-125$ & upper Pleistocene & 489 & 10.21 & 85.08 & 0.01 & 7 & 0.07 & 1.4 & - & -26.8 & - & 23 & 40 \\
\hline Hole & 445, Unit & & & & & & & & & & & & & & \\
\hline 60 & 3 & $100-125$ & upper Eocene & 563.5 & 5.15 & 42.93 & 0.12 & 8 & 0.69 & 1.8 & -26.0 & -27.2 & +1.2 & 36 & 7 \\
\hline Hole & 445, Unit & & & & & & & & & & & & & & \\
\hline 65 & 2 & $100-125$ & upper Eocene & 610 & 0.14 & 1.16 & 0.17 & 11 & 0.81 & 1.5 & -25.3 & -27.1 & +1.8 & 81 & 2 \\
\hline 65 & 2 & $125-150$ & upper Eocene & 611 & 0.33 & 2.73 & 0.14 & 4 & 0.26 & 1.9 & - & -27.0 & - & 7 & 34 \\
\hline Hole & 445, Unit & & & & & & & & & & & & & & \\
\hline 75 & 4 & $100-123$ & middle Eocene & 706 & 2.49 & 20.72 & 0.05 & 3 & 0.59 & 2.6 & - & -28.3 & - & 38 & 45 \\
\hline 75 & 4 & $123-145$ & middle Eocene & 708 & 2.74 & 22.86 & 0.06 & 2 & 0.33 & 1.5 & - & -29.9 & - & 14 & 97 \\
\hline 80 & 4 & $100-125$ & middle Eocene & 753 & 1.37 & 11.43 & 0.19 & 2 & 0.10 & 2.2 & - & -25.6 & - & 0 & 41 \\
\hline 80 & 4 & $125-150$ & middle Eocene & 754.5 & 1.72 & 14.31 & 0.12 & 2 & 0.16 & 1.6 & - & -27.0 & - & 10 & 61 \\
\hline 83 & 3 & $100-125$ & middle Eocene & 781 & 0.82 & 6.83 & 0.23 & 10 & 0.44 & 1.4 & -26.6 & -26.2 & -0.4 & 32 & 6 \\
\hline 83 & 3 & $125-150$ & middle Eocene & 782.5 & 0.69 & 5.76 & 0.18 & 3 & 0.16 & 1.0 & - & -27.5 & - & 31 & 52 \\
\hline & & & & & & Daito & Basin sout: & of the Da: & to Ridge & & & & & & \\
\hline Hole & 446, Unit & & & & & & & & & & & & & & \\
\hline 3 & 5 & $90-115$ & Miocene & 15 & 0.21 & 1.75 & 0.21 & 4 & 0.19 & 3.0 & - & - & - & 32 & 467 \\
\hline 9 & 4 & $100-125$ & upper-lower Miocene & 73.5 & 0.06 & 0.50 & 0.13 & 10 & 0.79 & 2.0 & -25.5 & -26.9 & +1.4 & 17 & 238 \\
\hline 14 & 4 & $94-119$ & middle Oligocene & 121 & 0.01 & 0.08 & 0.24 & 19 & 0.80 & 1.1 & -26.6 & -27.6 & +1.0 & & 138 \\
\hline Hole & 446, Unit & III, Sub-un & t IIIA & & & & & & & & & & & & \\
\hline 24 & 2 & $100-125$ & middle Eocene & 213 & 0.08 & 0.67 & 0.13 & 9 & 1.08 & 1.3 & -26.3 & -28.6 & +2.3 & 32 & 6 \\
\hline 30 & 5 & $90-115$ & lower-middle Eocene & 273 & 0.12 & 1.00 & 0.13 & 11 & 0.89 & 1.1 & -26.3 & -29.8 & +3.5 & 18 & 11 \\
\hline 34 & 5 & $90-115$ & lower-middle Eocene & 312 & 0.07 & 0.58 & 0.15 & 11 & 1.57 & 1.2 & -26.2 & -28.9 & +2.7 & 25 & 3 \\
\hline Hole & 446. Unit & & & & & & & & & & & & & & \\
\hline 41 & 2 & $90-115$ & lower-middle Eocene & 273 & 0.81 & 6.75 & 0.14 & 5 & 0.35 & 1.7 & - & -29.8 & - & 24 & 2 \\
\hline Hole & $446 \mathrm{~A}, \mathrm{Un}$ & it IV & & & & & & & & & & & & & \\
\hline 10 & 2 & $100-125$ & upper-lower Eocene & 489 & 0.07 & 0.55 & 0.11 & 5 & 0.42 & 1.1 & - & - & - & 24 & 19 \\
\hline & & & & & & Eastern sid & de of the $\mathrm{W}$ & st Philipp & e Basin & & & & & & \\
\hline Leg 59 & & & & & & & & & & & & & & & \\
\hline Hole & 447A, Un & it III & & & & & & & & & & & & & \\
\hline 6 & 3 & $100-125$ & early-upper Oligocene & 50.5 & 0.30 & 2.53 & 0.27 & 17 & 0.64 & 1.0 & -26.4 & -28.6 & +2.2 & 19 & 43 \\
\hline 6 & 3 & $125-150$ & early-upper Oligocene & 51.5 & 0.37 & 3.06 & 0.17 & 6 & 0.32 & 1.0 & - & -28.7 & - & 24 & 25 \\
\hline
\end{tabular}


Table 1. (Continued).

\begin{tabular}{|c|c|c|c|c|c|c|c|c|c|c|c|c|c|c|c|c|}
\hline \multirow[b]{2}{*}{ Core } & \multirow[b]{2}{*}{ Section } & \multirow[b]{2}{*}{$\begin{array}{l}\text { Interval } \\
(\mathrm{cm})\end{array}$} & \multirow[b]{2}{*}{ Chronostratigraphy } & \multirow[b]{2}{*}{$\begin{array}{l}\text { Sub-bottom } \\
\text { Depth }(\mathrm{m})\end{array}$} & \multicolumn{2}{|c|}{ Carbonate } & \multicolumn{2}{|c|}{ Organic Carbon } & \multirow[b]{2}{*}{ 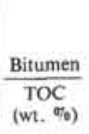 } & \multirow[t]{2}{*}{$\overline{O E P}$} & \multicolumn{3}{|c|}{$\begin{array}{c}\text { Carbon Isotepic } \\
\text { Composition }\left(\delta^{13} \mathrm{C}_{P D B}\right)\end{array}$} & \multicolumn{2}{|c|}{$\begin{array}{c}\text { Kjeldalh } \\
\text { Nitrogen (ppm) }\end{array}$} & \multirow{2}{*}{$\begin{array}{c}\begin{array}{c}\text { Atomic } \\
\text { Ratio }\end{array} \\
\text { Organic C } \\
\text { Organic N }\end{array}$} \\
\hline & & & & & $\begin{array}{l}\text { Carbon } \\
\text { (wt. \%) }\end{array}$ & $\begin{array}{c}\text { As } \\
\mathrm{CaCO}_{3} \\
\left(\mathrm{wt}, \%_{0}\right)\end{array}$ & $\begin{array}{l}\text { Total } \\
\text { (wt. \%) }\end{array}$ & $\begin{array}{l}\text { Bitumen } \\
\text { (ppm) }\end{array}$ & & & Bitumen & Kerogen & $\begin{array}{l}\text { Diff. } \\
B-K\end{array}$ & $\begin{array}{l}\text { Ammonium } \\
\text { Nitrogen } \\
\text { (half) }\end{array}$ & $\begin{array}{l}\text { Organic N } \\
\text { (full-half) }\end{array}$ & \\
\hline \multirow{2}{*}{\multicolumn{17}{|c|}{ Western edge of the Palau-Kyushu Ridge }} \\
\hline Hole & 448, Unit & & & & & & & & & & & & & & & \\
\hline 2 & 5 & $100-125$ & lower Miocene & 12 & 8.70 & 72.49 & 0.14 & 19 & 1.37 & 1.3 & -24.2 & -27.0 & +2.8 & 28 & 39 & 42 \\
\hline 8 & 2 & $100-125$ & lower Miocene & 64.5 & 9.84 & 82.00 & 0.12 & 10 & 0.88 & 1.1 & -23.6 & -26.5 & +2.9 & 23 & 19 & 74 \\
\hline \multicolumn{17}{|c|}{ Hole 448 , Unit 11} \\
\hline 13 & 5 & $100-125$ & upper Oligocene & 116.5 & 10.14 & 84.53 & 0.08 & 8 & 0.99 & 1.8 & -25.4 & -27.5 & +2.1 & 25 & 9 & 104 \\
\hline 19 & 2 & $100-125$ & upper Oligocene & 169 & 5.31 & 44.24 & 0.10 & 10 & 1 & 1.5 & -30.7 & -27.4 & -3.3 & 27 & 59 & 20 \\
\hline \multicolumn{17}{|c|}{ Western side of the Parece Vela Basin } \\
\hline $\begin{array}{l}\text { Hole } \\
2\end{array}$ & 449 , Unit & ${ }^{1} 100-125$ & & 4 & 0.16 & 1.33 & 0.24 & 10 & 0.42 & 0.9 & -28.4 & -25.7 & 2.7 & 42 & 66 & 42 \\
\hline \multicolumn{17}{|c|}{ Hole 449 , Unit IV } \\
\hline 8 & 2 & $100-125$ & & 59.5 & 0.28 & 2.34 & 0.09 & 15 & 1.60 & 1.9 & - & -28.6 & - & 36 & 29 & 36 \\
\hline \multicolumn{17}{|c|}{ Hole 449, Unit $\mathrm{V}$} \\
\hline 13 & 4 & $100-125$ & lower Miocene & 110 & 7.22 & 60.15 & 0.22 & 3 & 0.13 & 1.1 & - & -27.0 & - & 27 & 34 & 76 \\
\hline \multirow{2}{*}{\multicolumn{17}{|c|}{ Eastern Side of the Parece Vela Basin }} \\
\hline Hole & 450, Unit & I, Sub-unit & & & & & & & & & & & & & & \\
\hline 2 & 5 & $100-125$ & Pliocene & 14.5 & 0.01 & 0.05 & 0.32 & 440 & 13.88 & 1.2 & - & - & - & 38 & 25 & 149 \\
\hline \multicolumn{17}{|c|}{ Hole 450, Unit II, Sub-unit IIA } \\
\hline 14 & 4 & $100-125$ & middle Miocene & 126 & 0.28 & 2.36 & 0.08 & 14 & 1.69 & 1.5 & - & -28.0 & - & 34 & 8 & 117 \\
\hline 14 & 4 & $125-150$ & middle Miocene & 127 & 0.58 & 4.82 & 0.001 & 9 & 0.90 & 1.1 & -26.1 & - & - & is & 26 & $<1$ \\
\hline 19 & 5 & $100-125$ & middle Miocene & 175 & 0.70 & 5.86 & 0.13 & 5 & 0.36 & 1.9 & - & -28.1 & - & 13 & 57 & 27 \\
\hline 19 & 5 & $125-150$ & middle Miocene & 176 & 0.66 & 5.50 & 0.18 & 9 & 0.13 & 1.3 & - & -28.1 & - & 21 & 33 & 64 \\
\hline 30 & 3 & $100-125$ & middle Miocene & 276 & 0.71 & 5.88 & 0.02 & 11 & 5.58 & 1.2 & - & -28.0 & - & 36 & 35 & 7 \\
\hline 30 & 3 & $125-150$ & middle Miocene & 277 & 6.15 & 51.27 & 0.09 & 35 & 4.07 & 1.7 & -27.9 & -28.2 & +0.3 & 18 & 81 & 13 \\
\hline \multicolumn{17}{|c|}{ Eastern edge of the West Mariana Ridge } \\
\hline $\begin{array}{l}\text { Hole } \\
2\end{array}$ & 451, Unit & ${ }^{1} 100-125$ & Quaternary & 12 & 0.21 & 1.75 & 0.27 & 15 & 0.55 & 1.6 & - & -27.4 & - & 20 & 110 & 29 \\
\hline \multicolumn{17}{|c|}{ Hole 45I, Unit II } \\
\hline 6 & 3 & $100-125$ & upper Miocene & 46 & 1.09 & 9.08 & 0.11 & 11 & 1.00 & 1.6 & - & -27.7 & - & 19 & - & - \\
\hline 6 & 3 & $125-150$ & upper Miocene & 47,3 & 2.63 & 21.91 & 0.03 & 4 & 1.06 & 1.4 & - & -27.6 & - & - & - & - \\
\hline
\end{tabular}

samples for which a bitumen value is reported, the averages of the $\delta^{13} C_{P D B}$ values for both the bitumen and kerogen are similar, -26.7 and -26.6 , respectively. In the past, $\delta^{13} C_{P D B}$ values for the bitumen fraction have been used to characterize organic source. The common interpretation of these $\delta^{13} C_{P D B}$ values, at least for deltaic environments, is that terrestrial, brackish, and marine-derived organic matter falls into the following ranges: less than $-30,-29$ to -30 , and greater than -29 , respectively. In open marine environments the classification scheme breaks down. Nevertheless, very light $\delta$-values $(<-29)$ are indicative of primarily terrigenous organic matter. Based on this designation, the four samples, 442A-28-2, 444-2-4, 444A-1-4, and 448-19-2, contain primarily terrigenously derived organic matter $\left(\delta^{13} \mathrm{C}_{P D B}=-30.7\right.$, respectively).

The $\delta^{13} C_{P D B}$ values of the 62 kerogen fractions averaged -26.6 with a range from -21.8 to -29.9 . Ten cores within this range contained kerogens with $\delta^{13} C_{P D B}$ values less than -25 . All 10 are from the Shikoku Basin and the small basin in the Daito Ridge.

The isotopic composition of both the bitumen and kerogen is similar, which is unusual if we assume that the bitumen derived primarily from the kerogen. The breakdown of kerogen during maturation involves the splitting of various chemical bonds. The results of empirical observation of kerogen degradation have been that the carbon of bitumen is isotopically lighter than the carbon of the associated kerogen by 1 to $4 \delta$ units (Tissot and Welte, 1978). The difference between the bitumen and kerogen (B-K) $\delta$-values is either small (less than -1 ) or positive for most of the cores. These small differences can be explained by the late stage of genesis (the catagenesis to metagenesis stage), a mixture of organic source material that is immature admixed with mature organic matter (in this case the immature organic matter is isotopically lighter than the mature), or by the fact that bitumen is derived from isotopically similar organic matter in which case isotopic fractionation did not occur during bitumen formation.

\section{Organic Nitrogen}

The organic nitrogen content determined by the Full minus Half Kjeldahl varies greatly within the samples. Generally, the organic nitrogen content is greater than $100 \mathrm{ppm}$ for Samples 442A, 442B, 443, 444, 444A, and 445. The nitrogen content in Hole 445 is less than 100 ppm below 441.5 meters to depth and for the remaining 36 cores, with the exception of the Miocene to Oligocene units in Hole 446.

The atomic ratio of organic carbon to organic nitrogen $(C / N)$ is used herein to reflect the organic nitrogen content of the sedimentary organic matter. Because the organic nitrogen is an approximation of the true value, the organic nitrogen values reported in Table 1 are only relative and are used here only to provide approximations of the organic nitrogen content. Values of $\mathrm{C} / \mathrm{N}$ near 1 are not realistic but do indicate a rather high concentration of nitrogen. $\mathrm{C} / \mathrm{N}$ of the sedimentary organic matter for the Shikoku Basin Holes 442A, 442B, 443, 444, and 444A and the Daito Ridge Basin Hole 445 down to the upper Oligocene at 489 meters is less than 11. Low values for this ratio are indicative of marine organic matter-that is, a high organic nitrogen content $(\mathrm{C} / \mathrm{N}<20)$ is associated with marine-derived organic matter. Conversely, a high ratio $(>20)$ is in- 
Table 2. A compilation of the ratio of pristane to phytane and the marine versus terrestrial $n$-alkane as given by the ratio $\left(n-\mathrm{C}_{21}+\right.$ $\left.n-\mathrm{C}_{22}\right) /\left(n-\mathrm{C}_{28}+n-\mathrm{C}_{29}\right)$.

\begin{tabular}{|c|c|c|c|c|c|c|c|}
\hline Core & Section & $\begin{array}{c}\text { Interval } \\
(\mathrm{cm})\end{array}$ & $\begin{array}{l}\text { Sub-bottom } \\
\text { Depth (m) }\end{array}$ & $\frac{\text { Pristane }}{\text { Phytane }}$ & $\frac{n \cdot \mathrm{C}_{21}+n-\mathrm{C}_{22}}{n-\mathrm{C}_{28}+n \cdot \mathrm{C}_{29}}$ & $\frac{\text { Pristane }}{\mathrm{C}-17}$ & $\frac{\text { Phytane }}{C-18}$ \\
\hline \multicolumn{8}{|c|}{ Hole 442A, Unit I } \\
\hline & 3 & 93-118 & 13.8 & 0.7 & 0.5 & 0.4 & 0.5 \\
\hline 7 & 3 & $100-125$ & 61.0 & 2.1 & 1.0 & 0.5 & 0.2 \\
\hline 14 & 2 & $100-125$ & 127.5 & 1.8 & 1.0 & 0.4 & 0.2 \\
\hline \multicolumn{8}{|c|}{ Hole 442A, Unit II, Sub-unit IIA } \\
\hline 19 & 2 & $100-125$ & 173.5 & 0.2 & 0.1 & 0.9 & 1.3 \\
\hline 19 & 2 & $125-150$ & 174.7 & 0.5 & 0.5 & 0.8 & 1.3 \\
\hline \multicolumn{8}{|c|}{ Hole 442A, Unir III, Sub-unit IIIA } \\
\hline 23 & 2 & $100-125$ & 211.6 & 0.9 & 1.7 & 0.2 & 0.2 \\
\hline 23 & 2 & $125-150$ & 213.0 & 1.2 & 1.4 & 0.4 & 0.1 \\
\hline 28 & 2 & $100-125$ & 259.0 & 0.7 & 0.7 & 1.2 & 0.4 \\
\hline 28 & & $125-150$ & 260.5 & 0.9 & 0.7 & 0.3 & 0.3 \\
\hline Hole 4 & 2B, Unit 1 & II. Sub-uni & HB & & & & \\
\hline & 3 & $100-125$ & 281 & 0.7 & 1.0 & 0.5 & 0.6 \\
\hline Hole 4 & 3. Unit IV & Sub-unit & VA & & & & \\
\hline 23 & 3 & $100-125$ & 210.5 & 0.4 & 0.4 & 0.3 & 0.5 \\
\hline 31 & 3 & $100-125$ & 291 & 0.9 & 0.6 & 0.5 & 0.5 \\
\hline 34 & & $100-125$ & 318 & - & - & - & - \\
\hline Hole 4 & 4, Unit 1 . & Sub-unit IA & & & & & \\
\hline & 4 & $94-119$ & 11.5 & 1.1 & 1.2 & 0.6 & 0.4 \\
\hline Hole 4 & 4. Unit II & & & & & & \\
\hline 7 & 5 & $94-119$ & 60.5 & 0.9 & 0.4 & 0.4 & 0.4 \\
\hline Hole 44 & 4A, Unit & III, Sub-uni & IIIA & & & & \\
\hline 1 & 4 & $100-125$ & 87.5 & 0.7 & 0.4 & 0.3 & 0.4 \\
\hline Hole 4 & $4 \mathrm{~A}$, Unit 1 & III, Sub-uni & IIIB & & & & \\
\hline 6 & 4 & $71-96$ & 134.7 & 0.9 & 0.5 & 0.3 & 0.3 \\
\hline 9 & 3 & $90-115$ & 162 & 0.6 & 0.9 & 0.6 & 0.5 \\
\hline Hole 44 & 4A, Unit I & IV, Sub-unit & IVB & & & & \\
\hline 14 & 3 & $100-125$ & 209.3 & 0.3 & 1.1 & 0.4 & 1.0 \\
\hline Hole 44 & $4 \mathrm{~A}$, Unit I & V. Sub-uni & IVC & & & & \\
\hline 22 & 5 & $100-125$ & 269.3 & 0.6 & 1.0 & 0.5 & 0.6 \\
\hline Hole 44 & 5. Unit 1 . & Sub-unit LA & & & & & \\
\hline 1 & 4 & $94-119$ & 4.0 & 0.7 & 0.8 & 0.7 & 0.6 \\
\hline 1 & 4 & $119-144$ & 5.5 & 0.6 & 1.0 & 0.6 & 0.6 \\
\hline 6 & 5 & $94-119$ & 53.5 & 0.3 & 0.2 & 0.5 & 1.5 \\
\hline Hole 44 & 5. Unit 1 . & Sub-unit IB & & & & & \\
\hline 11 & 2 & $100-125$ & 96.5 & 2.8 & 0.6 & 1.7 & 1.0 \\
\hline Hole 44 & 5, Unit II, & Sub-unit II & & & & & \\
\hline 17 & 1 & $90-115$ & 152 & 0.2 & 0.2 & 0.5 & 3.7 \\
\hline 23 & 4 & $0-25$ & 212.5 & 0.2 & 0.4 & 0.4 & 2.3 \\
\hline 27 & 4 & $94-119$ & 251.5 & 0.1 & 0.7 & 0,4 & 4.4 \\
\hline Hole 4 & 5. Unit II. & Sub-unit II & & & & & \\
\hline 32 & 2 & $90-115$ & 296 & 1.5 & 0.3 & 0.4 & 0.3 \\
\hline Hole 4 & 5. Unit II, & Sub-unit II & & & & & \\
\hline 37 & 4 & $90-115$ & 346 & 0.6 & 0.4 & 1.6 & 5.9 \\
\hline 42 & 5 & $100-125$ & 395 & 0.2 & 1.3 & 0.4 & 2.0 \\
\hline 47 & 4 & $100-125$ & 441.5 & 0.1 & 1.2 & 0.3 & 3.1 \\
\hline 52 & 4 & $100-125$ & 489 & 0.3 & 1.4 & 0.3 & 2.3 \\
\hline Hole 4 & 5. Unit Iil & & & & & & \\
\hline 60 & 3 & $100-125$ & 563.5 & 0.2 & 0.6 & 0.6 & 1.6 \\
\hline Hole 4 & 5. Unit IV & & & & & & \\
\hline 65 & 2 & $100-125$ & 610 & 0.6 & 0.4 & 0.5 & 0.8 \\
\hline 65 & & $125-150$ & 611 & 0.2 & 0.3 & 0.6 & 1.9 \\
\hline Hole 4 & 5. Unit $\mathrm{V}$ & & & & & & \\
\hline 75 & 4 & $100-123$ & 706 & 0.5 & 0.8 & 0.5 & 2.0 \\
\hline 75 & 4 & $123-145$ & 708 & 0.5 & 0.7 & 1.0 & 0.7 \\
\hline 80 & 4 & $100-125$ & 753 & 0.3 & 0.4 & 0.3 & 1.0 \\
\hline 80 & 4 & $125-150$ & 754.5 & 0.6 & 0.9 & 1.0 & 1.4 \\
\hline 83 & 3 & $100-125$ & 781 & 0.9 & 0.5 & 0.4 & 0.6 \\
\hline 83 & 3 & $125-150$ & 782.5 & 0.8 & 0.6 & 0.4 & 0.6 \\
\hline Hole 44 & 6. Unit II & & & & & & \\
\hline 3 & 5 & $90-115$ & 15 & 0.3 & 0.3 & 0.5 & 1.3 \\
\hline 3 & 4 & $100-125$ & 73.5 & 0.2 & 1.4 & 0.3 & 1.3 \\
\hline 14 & 4 & $94-119$ & 121 & 0.5 & 0.9 & 0.4 & 0.6 \\
\hline Hole 44 & 6. Unit III & Sub-unit I & IIA & & & & \\
\hline 24 & 2 & $100-125$ & 213 & 0.6 & 5.3 & 0.4 & 0.4 \\
\hline 30 & 5 & $90-115$ & 273 & 1.1 & 1.0 & 0.2 & 0.4 \\
\hline 34 & 5 & $90-115$ & 312 & 1.0 & 0.5 & 0.4 & 0.4 \\
\hline Hole 44 & 6. Unit IV & & & & & & \\
\hline 41 & 2 & $90-115$ & 273 & 0.3 & 1.6 & 0.6 & 1.9 \\
\hline Hole 44 & $6 \mathrm{~A}$, Unit I & & & & & & \\
\hline 10 & 2 & $100-125$ & 489 & 0.2 & 0.8 & 0.5 & 2.3 \\
\hline Hole 4 & 7A, Unit I & & & & & & \\
\hline 6 & 3 & $100-125$ & 50.5 & 0.7 & 3.4 & 0.5 & 0.7 \\
\hline 6 & 3 & $125-150$ & 51.5 & 0.4 & 0.6 & 0.5 & 1.4 \\
\hline Hole 44 & 8. Unit 1 & & & & & & \\
\hline 2 & 5 & $100-125$ & 12 & 0.4 & 0.6 & 1.0 & 0.8 \\
\hline 8 & 2 & $100-125$ & 64.5 & 0.8 & 1.3 & 0.6 & 0.4 \\
\hline Hole 44 & 8. Unit II & & & & & & \\
\hline 13 & 5 & $100-125$ & 116.5 & 0.5 & 1.0 & 0.5 & 0.7 \\
\hline 19 & 2 & $100-125$ & 169 & 0.9 & 1.9 & 0.4 & 0.4 \\
\hline Hole 44 & 9, Unit 1 & & & & & & \\
\hline 2 & 3 & $100-125$ & 4 & 0.6 & 2.4 & 2.6 & 2.3 \\
\hline
\end{tabular}

Table 2. (Continued).

\begin{tabular}{|c|c|c|c|c|c|c|c|}
\hline Core & Section & $\begin{array}{l}\text { Interval } \\
(\mathrm{cm})\end{array}$ & $\begin{array}{l}\text { Sub-bottom } \\
\text { Depth }(\mathrm{m})\end{array}$ & $\frac{\text { Pristane }}{\text { Phytane }}$ & $\frac{n-\mathrm{C}_{21}+n \cdot \mathrm{C}_{22}}{n \cdot \mathrm{C}_{28}+n \cdot \mathrm{C}_{29}}$ & $\frac{\text { Pristane }}{\mathrm{C}-17}$ & $\frac{\text { Phytane }}{\text { C.18 }}$ \\
\hline $\begin{array}{c}\text { Hole } \\
8\end{array}$ & $\begin{array}{l}\text { 49. Unit IV } \\
2\end{array}$ & $100-125$ & 59.5 & 0.4 & 0.8 & 0.4 & 0.9 \\
\hline $\begin{array}{l}\text { Hole } \\
13\end{array}$ & 49. Unit V & $100-125$ & 110 & 0.1 & 0.8 & 0.3 & 2.1 \\
\hline$\underset{2}{\mathrm{Hole}}$ & So, Unit 1. & $\begin{array}{l}\text { Sub-unit } 1 \\
100-125\end{array}$ & 14.5 & 0.8 & 1.3 & 0.7 & 0.5 \\
\hline $\begin{array}{c}\text { Hole } \\
14 \\
14 \\
19 \\
19 \\
30 \\
30\end{array}$ & $\begin{array}{c}\text { 50. Unit II. } \\
4 \\
4 \\
5 \\
5 \\
3 \\
3\end{array}$ & $\begin{array}{c}\text { Sub-unit } 1 \\
100-125 \\
125-150 \\
100-125 \\
125-150 \\
100-125 \\
125-150\end{array}$ & $\begin{array}{l}\text { IIA } \\
126 \\
127 \\
175 \\
176 \\
276 \\
277\end{array}$ & $\begin{array}{l}0.9 \\
0.4 \\
0.6 \\
0.3 \\
0.3 \\
0.3\end{array}$ & $\begin{array}{l}4.3 \\
0.6 \\
0.7 \\
0.8 \\
0.6 \\
1.2\end{array}$ & $\begin{array}{l}0.5 \\
0.5 \\
0.6 \\
0.4 \\
0.4 \\
0.5\end{array}$ & $\begin{array}{l}0.5 \\
1.1 \\
0.8 \\
1.0 \\
1.4 \\
1.6\end{array}$ \\
\hline $\mathrm{Hole}_{2}$ & 51, Unit I & $100-125$ & 12 & 0.5 & 1.2 & 0.5 & 0.9 \\
\hline & 51, Unit II & $100-125$ & 46 & & 1.5 & 0.4 & 0.8 \\
\hline $\begin{array}{l}6 \\
6\end{array}$ & 3 & $125-150$ & $\begin{array}{l}46 \\
47.3\end{array}$ & 0.5 & 1.0 & 0.5 & $\begin{array}{l}1.8 \\
1.0\end{array}$ \\
\hline
\end{tabular}

dicative of terrigenous organic matter. $\mathrm{C} / \mathrm{N}$ is highest in Hole 445 at 610 meters $(\mathrm{C} / \mathrm{N}=992)$. Generally $\mathrm{C} / \mathrm{N}$ is greater than 20 for the remaining cores. These high values reflect a higher terrigenous input into these sediments than is seen in other sediments in the area that we studied.

\section{Average Values for Organic Chemical Properties of Open Marine Sediments}

The averaged values of 11 chemical properties of open marine sediments are given in Table 3 . These sediments are from Site 436 on the outer trench slope of the Japan Trench (Schorno, in press a), each site reported herein, and seven sites from the Mariana Trough and Trench (Schorno, in press b). The closest agreement among the selected chemical properties of these open marine sediments among sites is noted in the ratio of pristane to phytane $(\mathrm{Pr} / \mathrm{Ph}=0.6 \mathrm{~S} . \mathrm{D} .=0.12)$, the percentage of organic carbon $(\mathrm{TOC}=0.14 \mathrm{~S}$.D. $=$ $0.04)$, the carbon isotopic composition of the bitumen $\left(\delta^{13} \mathrm{C}_{P D B}=-26.6 \mathrm{~S} . \mathrm{D} .=1.0\right)$, and the kerogen $\left(\delta^{13} C_{P D B}=-26.6\right.$ S.D. $\left.=1.8\right)$ fractions.

\section{CONCLUSIONS}

As noted herein, the standard techniques used to determine both organic source and degree of maturation must be re-evaluated when considering purely open marine sediments. The interpretations of the commonly used parameters $S$ and $\overline{O E P}$ are suspect for this particular type organic source. More specific source and maturation indicators are needed for these sediments. It is hoped that bio-markers can fill this void. Open marine sediments appear to have several chemical properties in common, but additional research is needed to substantiate this.

\section{REFERENCES}

Hunt, J. M., 1979. The source rock. Petroleum Geochemistry and Geology: San Francisco (W. H. Freeman), pp. 302-303.

Lijmbach, G. M., 1975. On the origin of petroleum. 9th World Petroleum Congress, Proc. (Vol. 2): London (Applied Science Publishers), 357-369.

Philippi, G. T., 1974. The influence of marine and terrestrial source material on the composition of petroleum. Geochim. Cosmochim. Acta, 36(6):947-966. 
Schorno, K. S., in press a. Geochemistry of carbon: Legs 56 and 57 , Deep Sea Drilling Project. In Scientific Party, Init. Repts. DSDP, 56, 57, Pt. 2: Washington (U.S. Govt. Printing Office). , in press b. Geochemistry of carbon: International Phase of Ocean Drilling Project Leg 60. In Hussong, D., Uyeda, S., et al., Init. Repts. DSDP, 60: Washington (U.S. Govt. Printing Office).

Powell, T. G., and McKirdy, D. M., 1973. The effect of source material, rock type and diagenesis on the $n$-alkane content of sediments. Geochim. Cosmochim. Acta, 37:623-633.

Scalan, R. S., and Smith, J. E., 1976. An improved measure of the odd-even predorninance of the normal alkanes of sediment extracts and petroleum. Geochim. Cosmochim. Acta, 34:611-620.

Tissot, B. P., and Welte, D. H., 1978. Petroleum Formation and Occurrence: New York (Springer Verlag).

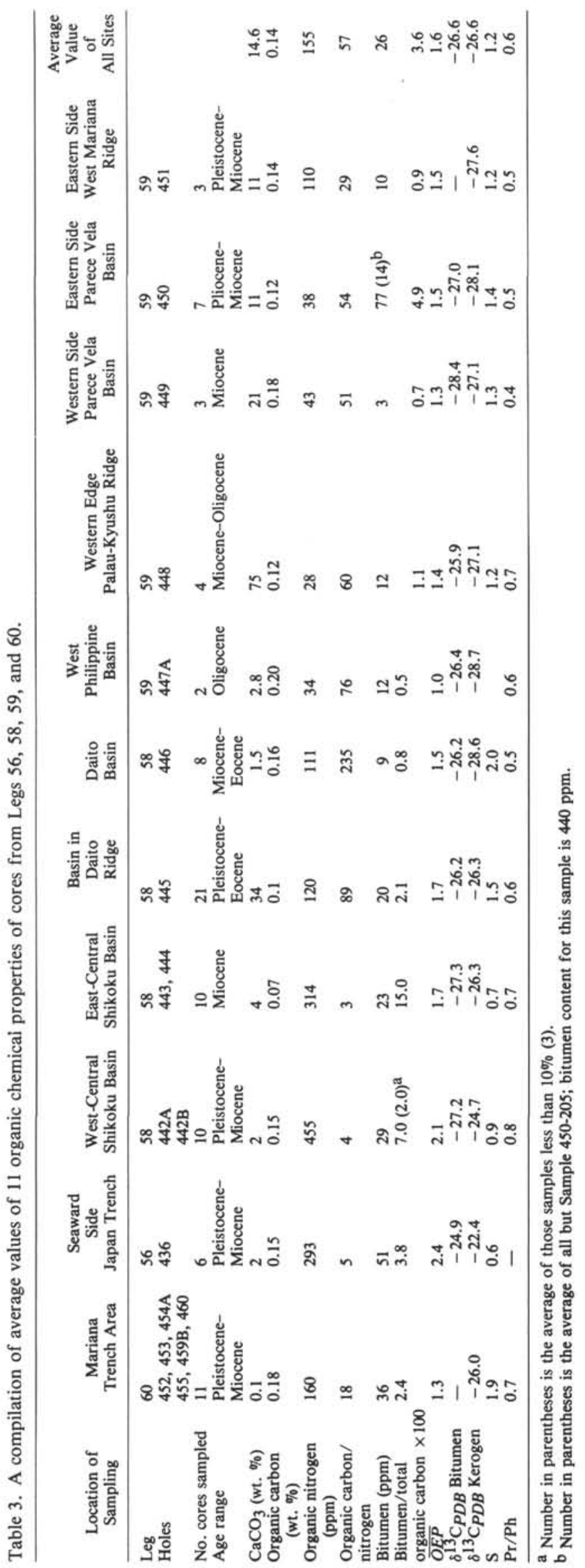

